\title{
Correction: A moderate role for cognitive models in agent-based modeling of cultural change
}

\section{Marshall Abrams}

Correspondence:

mabrams@uab.edu Department of Philosophy,

University of Alabama at Birmingham 900 13th Street South HB 414A Birmingham, AL 35294-1260, USA

(C2014 Abrams; licensee Springer. This is an Open Access article distributed under the terms of the Creative Commons

Attribution License (http://creativecommons.org/licenses/by/2.0), which permits unrestricted use, distribution, and reproduction in any medium, provided the original work is properly cited.

\section{Correction}

After publication of (Abrams 2013), I discovered errors in two formulas in the settle-nets paragraphs of the POPCO main loop section in Methods (page 13 in the PDF file). I correct these errors here. In the definition of the weighted sum $n_{i}$ of input activations $a_{j}$ from nodes $j$ to node $i$ across links with weights $w_{i j}$, the direction of the inequality was reversed. The definition of $n_{i}$ should read:

$$
n_{i}=\sum_{j} w_{i j} \max \left(0, a_{j}\right) \quad \text { for } w_{i j}<0 .
$$

There were also errors in the definition of $s_{i}$, the weighted sum of a node's current activation $a_{i}$ and its summed inputs $p_{i}$ and $n_{i}$ across positively and negatively weighted links, respectively. The definition of $s_{i}$ should read as follows:

$$
s_{i}=(1-.1) a_{i}+\left(.99-a_{i}\right) p_{i}+\left(a_{i}-(-.99)\right) n_{i}
$$

I regret these errors and hope that they do not cause confusion. Full source code for the version of POPCO used in Abrams (2013) can be found at https://github.com/mars0i/ popco.

Received: 3 December 2013 Accepted: 3 December 2013

Published: 06 Jan 2014

\section{References}

Abrams M: A moderate role for cognitive models in agent-based modeling of cultural change. Complex Adaptive Syst Model 2013, 1(16):1-33.

10.1186/2194-3206-2-1

Cite this article as: Abrams: Correction: A moderate role for cognitive models in agent-based modeling of cultural change. Complex Adaptive Systems Modeling 2014, 2:1 